Original Research Paper

\title{
The Human Facial Expression Classification Using the Center Kernel Subspace based the Ridge Regression
}

\author{
Arif Muntasa \\ Informatics Engineering, University of Trunojoyo, Madura, Indonesia
}

Article history

Received: 04-06-2015

Revised : 07-12-2015

Accepted: 21-12-2015

Email: arifmuntasa@if.trunojoyo.ac.id

\section{Introduction}

Many researchers have developed biometrics research field to improve the previous research. The results of the research have been implemented for many applications, such as motion detection, face detection, face recognition, finger recognition and palm recognition. However, the results of research are necessary to be improved, especially computational problem.

High dimensional of the training and the testing sets will effect on the high cost (Zhao and Pietikeinen, 2007; Tian et al., 2001). Therefore, it is necessary to conduct dimensionality reduction to the training and the testing sets.

Many algorithms have been implemented for dimensionality reduction, which are based on appearance (Shih et al., 2008; Wright et al., 2009; Martinez and Kak, 2001; Yang et al., 2004; Li and Yuan, 2005; Muntasa, 2014; 2015a; 2015b; Zhang and Zha, 2004; Sanguinetti, 2008), geometrical (Muntasa et al., 2012; Rizvandi et al., 2007; Cootes et al., 2000) and hybrid (Cootes et al., 2000; Tang and Wang, 2003).

Several cases based on appearance method often produce the singular matrix. If it occurred, the Eigenvalue and Eigenvector could not be determined.
Although, the singular value decomposition can be used to overcome it. However, appearance based also has another limitation, which produces the global features. In fact, the local characteristics are also necessary as the dominant feature of an object. Inability to separate nonlinearly features is also the weakness of an appearance-based method.

In this research, a kernel-based method is proposed. It is utilized to transform from input into the feature space. In this case, the center kernel is introduced. It is conducted to overcome inability appearance based method to separate random features. Furthermore, Gabor Filter Bank is utilized to normalize the object shifting by using five scales and eight orientations.

To reduce the high dimensionality, Two-Dimensional Fast Fourier Transform is proposed to overcome it. The results of dimensionality reduction as primary features, they will be measured by using Mahalanobis method.

Furthermore, the remaining of this paper will be organized as follows. Section II consists of the proposed method. The similarity measurement using Mahalanobis method is written in section III. Finally, the experimental results have been reported and analyzed in section IV. The last section contains conclusion of experimental results. 


\section{Research Method}

In this proposed method, an image is represented by using $f(x, y)$. In this case, $x$ and $y$ variables represent an axis and an ordinate position, whereas $f$ depicts the gray scale of an image. In general, it can be mathematically written:

$$
\begin{aligned}
& f(x, y) \mid x \in\{1,2,3, \cdots, H\}, \\
& y \in\{1,2,3, \cdots, W\}, \\
& f(x, y) \in\{1,2,3, \cdots, 255\}
\end{aligned}
$$

Furthermore, the original image as Equation (1) is processed by using five scales and eight orientations as seen in the Gabor Filter equation:

$$
\begin{aligned}
& \mathrm{g}(\mathrm{x}, \mathrm{y} ; \lambda, \theta, \psi, \sigma, \lambda)=\exp (\beta) \exp (\mathrm{i} \chi) \\
& \mathrm{g}(\mathrm{x}, \mathrm{y} ; \lambda, \theta, \psi, \sigma, \lambda)=\exp (\beta) \cos (\chi) \\
& \mathrm{g}(\mathrm{x}, \mathrm{y} ; \lambda, \theta, \psi, \sigma, \lambda)=\exp (\beta) \sin (\chi)
\end{aligned}
$$

The $\beta$ and $\chi$ values represent mathematics equation as seen follows:

$$
\begin{aligned}
& \beta=-\frac{\left(x^{\prime}\right)^{2}+\gamma^{2}\left(y^{\prime}\right)^{2}}{2 \sigma^{2}} \\
& \chi=\left(2 \pi \frac{x^{\prime}}{\lambda}+\psi\right)
\end{aligned}
$$

The values of $x^{\prime}$ and $y^{\prime}$ can be explained in the following equation:

$$
\begin{aligned}
& x^{\prime}=x \cos \theta+y \sin \theta \\
& y^{\prime}=-x \sin \theta+y \cos \theta
\end{aligned}
$$

Figure 1 represents the real part and Fig. 2 depicts the imaginary part by using five scales and eight orientation of the Gabor Filter.

Furthermore, the results of the Gabor Filter will be utilized as image input on the Two-Dimensional Fourier Transform as shown on the following equation:

$$
F(k x, k y)=\sum_{j x=1}^{n y-1} \sum_{j y=1}^{n x-1} e^{-i \theta} * f(j x, j y)
$$

In this case, the value of $\theta$ can be written as follows:

$$
\theta=\frac{2 \pi}{n x} * k x * j x+\frac{2 \pi}{n y} * k y * j y
$$

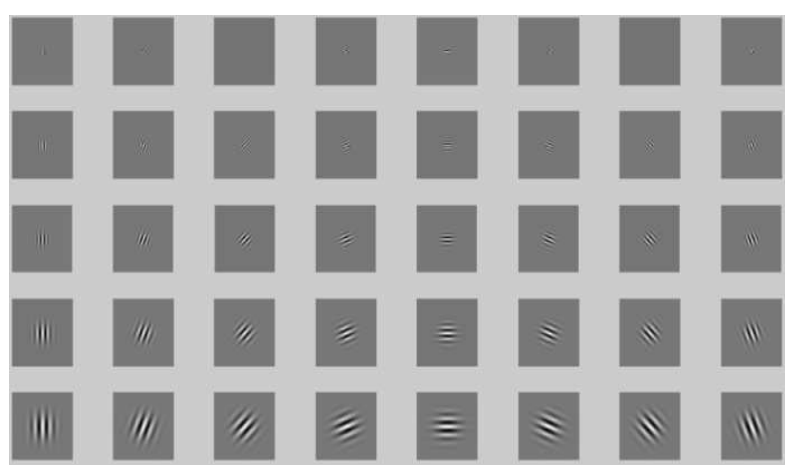

Fig. 1. The real part of Gabor filter

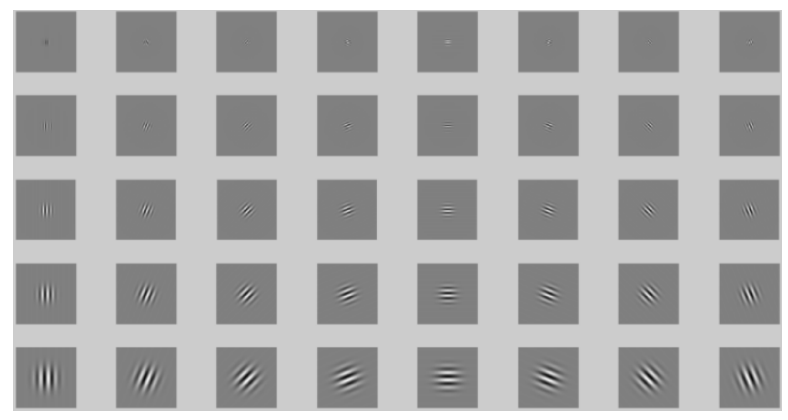

Fig. 2. The imaginary part of Gabor filter

To obtain the center kernel value, necessary to use the kernel center by using the following equation:

$$
\begin{aligned}
& K_{\text {centre }}=K-I_{3} * K-K * I_{3}+I_{3} * K * I_{3} \\
& I_{3}=I . * H_{I}
\end{aligned}
$$

Variable of $K$ depicts the polynomial kernel of Equation 11. The identity matrix and its height are represented by using $I$ and $H$. To obtain the ridge regression value; it is necessary to calculate the values of $W$ and $\varsigma$ as shown in equation:

$R_{r}=\frac{K_{\text {centre }} * K_{\text {centre }}+\zeta * I}{K_{\text {centre }} * W^{*} K_{\text {centre }}}$

The value of $W$ and $\varsigma$ depict the auxiliary function and the small value of the ridge regression as seen on the following function:

$\zeta=10^{-\min \left(\mathrm{K}_{\text {centro }}\right)}$

The Eigenvalue $(\lambda)$ and vector $(\Lambda)$ can be computed by using singular value decomposition based on Equation 13 and 14. The training features can be obtained by multiplication between square root of the Eigenvalue and the center kernel: 


$$
\begin{aligned}
& \text { Fea }_{\text {tran }}=\alpha * K_{\text {centre }} \\
& \alpha=\sqrt{\sum_{\mathrm{i}=1}^{\mathrm{n}} \lambda_{\mathrm{i}}}
\end{aligned}
$$

To obtain the testing features, it is necessary to calculate the value of $K_{c i}$ :

$$
\begin{aligned}
& K_{c t}=K-\left(I_{3} *\left(I_{3}\right)^{T}\right) * T_{f}-v \\
& v=\frac{K}{\alpha} *\left(I_{3} *\left(I_{3}\right)^{T}\right)+\left(I_{3} *\left(I_{3}\right)^{T}\right) * K^{*}\left(I_{3} *\left(I_{3}\right)^{T}\right)
\end{aligned}
$$

The testing feature can be modeled simplify as seen in the following:

$$
F e a_{\text {test }}=\alpha * K
$$

\section{Similarity Measurement Using Mahalanobis}

Furthermore, the results of feature extraction will be measured between testing object and training object. In this case, the Mahalanobis method is used to obtain the biggest probability as shown:

$$
d=\sqrt{\left(\left(\bar{X}_{j}-\bar{Y}_{j}\right) C_{j, j}^{-1}\left(\bar{X}_{j}-\bar{Y}_{j}\right)^{T}\right)}
$$

The value of $C$ represents $X$ and $Y$ covariance as seen on the following equation:

$$
\begin{aligned}
& C_{j, j}=\frac{m}{m+p}\left(C X_{j, j}\right)+\frac{p}{m+p}\left(C Y_{j, j}\right) \\
& C Y_{j, j}=\left(Y_{i, j}-\bar{Y}_{j}\right)^{T}\left(Y_{i, j}-\bar{Y}_{j}\right) \\
& C X_{j, j}=\left(X_{i, j}-\bar{X}_{j}\right)^{T}\left(X_{i, j}-\bar{X}_{j}\right)
\end{aligned}
$$

\section{Experimental Results and Analysis}

One of the face image databases for facial expression recognition is the Japanese Female Facial Expression or well known as the JAFFE. The proposed method was evaluated by using the JAFFE image database as the experimental data set. It has captured 213 times (Lyons et al., 1998), it consists of seven expressions. Distribution of the expression of JAFFE face image database can be seen in Table 1. A sample of JAFFE face image database can be shown in Fig. 3.

In this research, the proposed method has been evaluated by conducting the six times experiment.

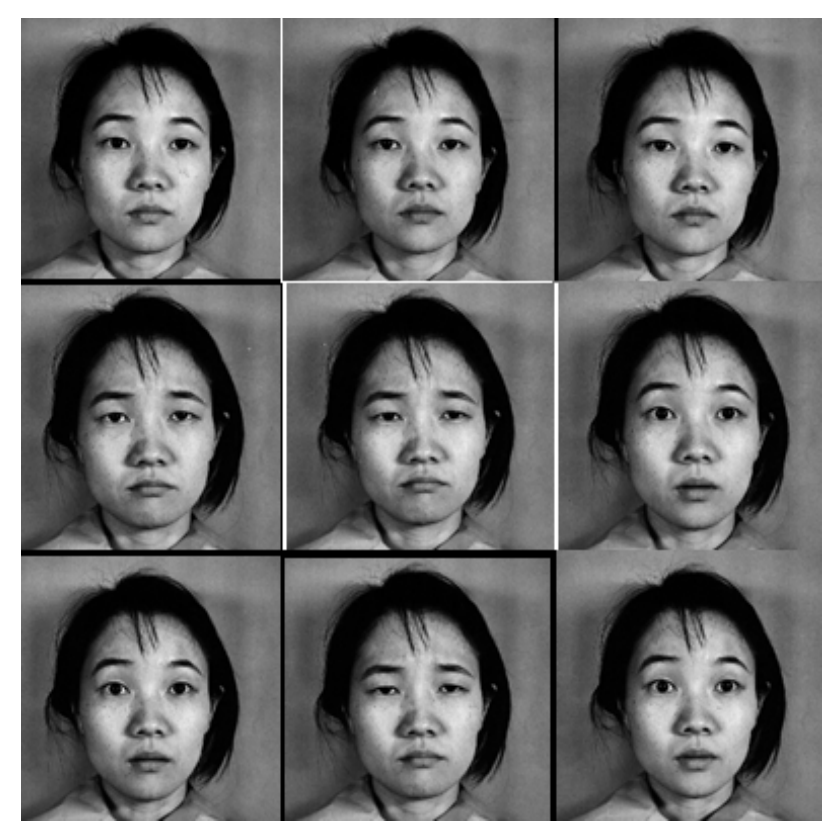

Fig. 3. Sample of the Facial Expression from JAFEE

Table 1. The JAFFE face image database distribution

\begin{tabular}{ll}
\hline Expression & Number of image sets \\
\hline Neutral & 30 \\
Happiness & 31 \\
Sadness & 31 \\
Surprise & 30 \\
Anger & 30 \\
Disgust & 29 \\
Fear & 32 \\
\hline
\end{tabular}

Table 2. Experimental results of the first scenario

\begin{tabular}{llll}
\hline Expression & $\begin{array}{l}\text { Number of } \\
\text { training set }\end{array}$ & $\begin{array}{l}\text { Number of } \\
\text { testing set }\end{array}$ & $\begin{array}{l}\text { Recognition } \\
\text { rate (\%) }\end{array}$ \\
\hline Neutral & 12 & 18 & 83.33 \\
Happiness & 13 & 18 & 88.89 \\
Sadness & 13 & 18 & 83.33 \\
Surprise & 12 & 18 & 83.33 \\
Anger & 12 & 18 & 83.33 \\
Disgust & 11 & 18 & 83.33 \\
Fear & 14 & 18 & 77.78 \\
Recognition average $(\%)$ & & 83.33 \\
\hline
\end{tabular}

Because every expression has some distribution difference of the data sets, a lot of training sets for each expression is also different. The experimental results for each scenario can be seen in Table 2-7.

On the first experiment, the testing set used is 18 images for each expression, whereas the remaining of them is used as training sets. The experimental results show that the highest error occurred on the fear expression image, which is four times error. The highest recognition rate occurred on a happiness expression 
image as seen in Table 2. On the first experiment, the recognition average obtained is $83.33 \%$

Table 3 demonstrated the experimental results of the second scenario. Seventeen images were used as the testing sets and the remaining of them were used as the training sets. The experimental results show that the lowest recognition rate has occurred on the fear image expression, fourteen images were correct recognized. The highest recognition is occurred on the neutral, anger and sadness expressions, which are sixteen of eighteen images. The recognition average of the second experiment is 84.03 .

On the third scenario, some training sets have been increased one for each expression so that the number of testing sets also decreases one. Table 4 shows that the additional of the training sets has increased the recognition average, though, on the certain expressions. The recognition rate of the third experiment is 86.61 . The recognition rate is also better than the first and the second experiment results.

The similar condition is also occurred on the fourth, fifth and sixth scenarios as seen in Table 5. The experimental results have also shown the improvement of recognition average. It is proportional to the increasing of the training sets used.

On the fifth scenario, the experimental results show that recognition rate almost has not increased. Its difference is only $0.01 \%$. In detail, the experimental results of the fifth scenario can be seen in Table 6 .

Table 3. Experimental results of the second scenario

\begin{tabular}{llll}
\hline Expression & $\begin{array}{l}\text { Number of } \\
\text { training set }\end{array}$ & $\begin{array}{l}\text { Number of } \\
\text { testing set }\end{array}$ & $\begin{array}{l}\text { Recognition } \\
\text { rate (\%) }\end{array}$ \\
\hline Neutral & 13 & 17 & 88.24 \\
Happiness & 14 & 17 & 82.35 \\
Sadness & 14 & 17 & 88.24 \\
Surprise & 13 & 17 & 82.35 \\
Anger & 13 & 17 & 88.24 \\
Disgust & 12 & 17 & 82.35 \\
Fear & 15 & 17 & 76.47 \\
Recognition average (\%) & & 84.03 \\
\hline
\end{tabular}

Table 4. Experimental results of the third scenario

\begin{tabular}{llll}
\hline Expression & $\begin{array}{l}\text { Number of } \\
\text { training set }\end{array}$ & $\begin{array}{l}\text { Number of } \\
\text { testing set }\end{array}$ & $\begin{array}{l}\text { Recognition } \\
\text { rate (\%) }\end{array}$ \\
\hline Neutral & 14 & 16 & 93.75 \\
Happiness & 15 & 16 & 87.50 \\
Sadness & 15 & 16 & 93.75 \\
Surprise & 14 & 16 & 81.25 \\
Anger & 14 & 16 & 87.50 \\
Disgust & 13 & 16 & 81.25 \\
Fear & 16 & 16 & 81.25 \\
Recognition average $(\%)$ & & 86.61 \\
\hline
\end{tabular}

Table 7 explained the experimental results of the last scenario. On the last scenario, the proposed method produced the recognition average $89.79 \%$. The increasing of recognition rate is $2.55 \%$ from the fifth scenario. It shows that the more number of training sets used, the more recognition average achieved.

Based on the first until the last scenario, the experimental results can be shown that the number of training sets used is proportional increasing of recognition average as seen in Fig. 4. Increment of the significant recognition average is not found for all experiments, but the increment of the recognition average is occurred for each experimental. The usage of the training sets has influenced recognition average obtained.

The experimental results were also compared with another paper, which is Local Binary Patterns (Ojala et al., 2002). The Local Binary Patterns method has produced $85.57 \%$. It shows that the Local Binary Pattern has outperformed to the proposed method for the first and the second scenarios. But the proposed method has outperformed for the third, the fourth, the fifth and the sixth scenarios.

Table 5. Experimental results of the fourth scenario

\begin{tabular}{llll}
\hline Expression & $\begin{array}{l}\text { Number of } \\
\text { training set }\end{array}$ & $\begin{array}{l}\text { Number of } \\
\text { testing set }\end{array}$ & $\begin{array}{l}\text { Recognition } \\
\text { rate } \mathbf{( \% )}\end{array}$ \\
\hline Neutral & 16 & 14 & 92.86 \\
Happiness & 16 & 15 & 86.67 \\
Sadness & 16 & 15 & 86.67 \\
Surprise & 16 & 14 & 92.86 \\
Anger & 16 & 14 & 85.71 \\
Disgust & 16 & 13 & 84.62 \\
Fear & 16 & 16 & 81.25 \\
Recognition average $\mathbf{( \% )}$ & & 87.23 \\
\hline
\end{tabular}

Table 6. Experimental results of the fifth scenario

\begin{tabular}{llll}
\hline Expression & $\begin{array}{l}\text { Number of } \\
\text { training set }\end{array}$ & $\begin{array}{l}\text { Number of } \\
\text { testing set }\end{array}$ & $\begin{array}{l}\text { Recognition } \\
\text { rate }(\%)\end{array}$ \\
\hline Neutral & 17 & 13 & 92.31 \\
Happiness & 17 & 14 & 85.71 \\
Sadness & 17 & 14 & 85.71 \\
Surprise & 17 & 13 & 92.31 \\
Anger & 17 & 13 & 84.62 \\
Disgust & 17 & 12 & 83.33 \\
Fear & 17 & 15 & 86.67 \\
Recognition average (\%) & & 87.24 \\
\hline
\end{tabular}

Table 7. Experimental results of the sixth scenario

\begin{tabular}{llll}
\hline Expression & $\begin{array}{l}\text { Number of } \\
\text { training set }\end{array}$ & $\begin{array}{l}\text { Number of } \\
\text { testing set }\end{array}$ & $\begin{array}{l}\text { Recognition } \\
\text { rate }(\%)\end{array}$ \\
\hline Neutral & 18 & 12 & 91.67 \\
Happiness & 18 & 13 & 84.62 \\
Sadness & 18 & 13 & 92.31 \\
Surprise & 18 & 12 & 91.67 \\
Anger & 18 & 12 & 91.67 \\
Disgust & 18 & 11 & 90.91 \\
Fear & 18 & 14 & 85.71 \\
Recognition average $(\%)$ & & 89.79 \\
\hline
\end{tabular}




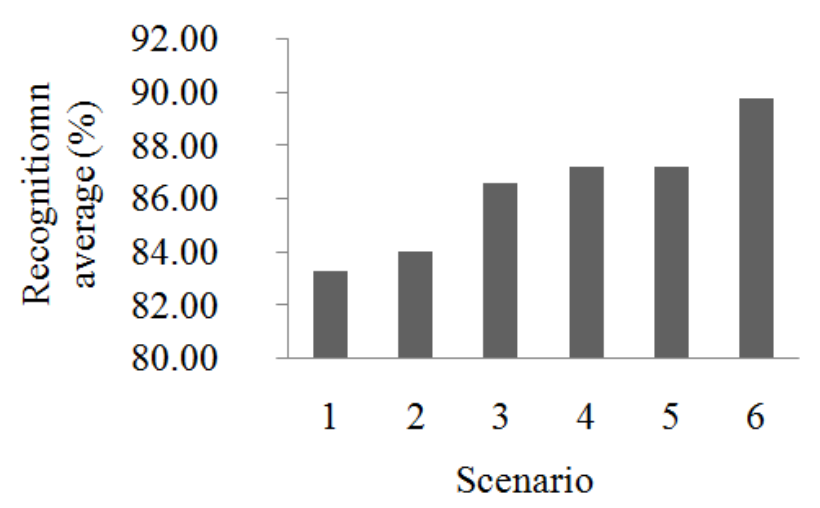

Fig. 4. The recognition average of proposed method

\section{Conclusion}

The proposed method, the center kernel subspacebased the ridge regression can be used as feature extraction for facial expression recognition. The experimental results show that the more training set used, the higher recognition rate was produced. It shows that the results of recognition depend on the training set used. However, there are some images misclassifications. They are caused by the similarity between the testing and the training images, where the similar images are not in a class. Misclassification can be improved by conducting the collaboration of method.

\section{Acknowledgement}

The author would like to thanks to peer researchers for improvement of the analysis, the detailed comment, for both contents and grammatical.

\section{Funding Information}

This research was support by Computational Artificial Intelligence Laboratory, Informatics Department, University of Trunojoyo, Madura, Indonesia.

\section{Ethics}

The paper is the original research results of the author. It did not contain plagiarism contents. This paper did not also published by another publisher.

\section{References}

Cootes, T.F., G.V. Wheeler, K.N. Walker and C.J. Taylor, 2000. Coupled-view active appearance models. Proceedings of the British Machine Vision Conference, (MVC' 00), BMVA Press, pp: 52-61. DOI: $10.5244 / C .14 .6$

Li, M. and B. Yuan, 2005. 2D-LDA: A statistical linear discriminant analysis for image matrix. Patt. Recognit. Lett., 26: 527-532.

DOI: $10.1016 /$ j.patrec.2004.09.007
Lyons, M., S. Akamatsu, M. Kamachi and J. Gyoba, 1998. Coding facial expressions with Gabor wavelets. Proceedings of the 3rd IEEE International Conference on Automatic Face and Gesture Recognition, Apr. 14-16, IEEE Xplore Press, Nara, Japan, pp: 200-205.

DOI: 10.1109/AFGR.1998.670949

Martinez, A.M. and A.C. Kak, 2001. PCA versus LDA. IEEE Trans. Patt. Analysis Machine Intellig., 23: 228-233. DOI: $10.1109 / 34.908974$

Muntasa, A., 2014. New modelling of modified two dimensional fisher face based feature extraction. TELKOMNIKA, 12: 115-122.

DOI: $10.12928 /$ telkomnika.v12i1.20

Muntasa, A., M.K. Shopan, M.H. Purnomo and K. Kunio, 2012. Enhancement of the adaptive shape variants average values by using eight movement directions for multi-features detection of facial sketch. ITB J. Inform. Commun. Technol., 6: 1-20. DOI: 10.5614/itbj.ict.2012.6.1.1

Muntasa, A., 2015a. Facial recognition using square diagonal matrix based on two-dimensional linear discriminant analysis. Int. Rev. Comput. Software (I.RE.CO.S.), 10: 718-725.

DOI: 10.15866/irecos.v10i7.6623

Muntasa, A., 2015b. A new approach: The local feature extraction based on the new regulation of the locally preserving projection, Applied Math. Sci., 9: 5065-5078. DOI: 10.12988/ams.2015.55408

Ojala, T., M. Pietikainen and T. Maenpaa, 2002. Multiresolution gray-scale and rotation invariant texture classification with local binary patterns. IEEE Trans. Patt. Analysis Machine Intellig., 24: 971-987. DOI: 10.1109/TPAMI.2002.1017623

Rizvandi, N.B, A. Pižurica and W. Philips, 2007. Deformable shape description using Active Shape Model (ASM). Proceedings of the 18th ProRISC Workshop on Circuits, Systems and Signal Processing (SSP' 07), Veldhoven, pp: 191-196.

Sanguinetti, G., 2008. Dimensionality reduction of clustered data sets. IEEE Trans. Patt. Analysis Machine Intellig., 30: 535-540. DOI: 10.1109/TPAMI.2007.70819

Shih, F.Y., C.F. Chuang and P.S.P. Wang, 2008. Performance comparisons of facial expression recognition in Jaffe database. Int. J. Patt. Recognit. Artificial Intellig., 22: 445-445. DOI: $10.1142 / \mathrm{S} 0218001408006284$

Tang, X. and X. Wang, 2003. Face sketch synthesis and recognition. Proceedings of the 9th IEEE International Conference on Computer Vision, Oct. 13-16, IEEE Xplore Press, Nice, France, pp: 687-694. DOI: 10.1109/ICCV.2003.1238414

Tian, Y., T. Kanade and J. Cohn, 2001. Recognizing action units for facial expression analysis. IEEE Trans. Patt. Analysis Machine Intellig., 23: 97-115. DOI: $10.1109 / 34.908962$ 
Wright, J., A.Y. Yang, A. Ganesh, S.S. Sastry and Y. Ma, 2009. Robust face recognition via sparse representation. IEEE Trans. Patt. Analysis Machine Intellig., 31: 210-227.

DOI: 10.1109/TPAMI.2008.79

Yang, J., D. Zhang, A.F. Frangi and J.Y. Yang, 2004. Two-dimensional PCA: A new approach to appearance-based face representation and recognition. IEEE Trans. Patt. Analysis Machine Intellig., 26: 131-137. DOI: 10.1109/TPAMI.2004.1261097
Zhang, Z. and H. Zha, 2004. Principal manifolds and nonlinear dimensionality reduction via local tangent space alignment. SIAM J. Sci. Comput., 26: 313-338. DOI: 10.1137/S1064827502419154

Zhao, G. and M. Pietikeinen, 2007. Dynamic texture recognition using local binary patterns with an application to facial expressions. IEEE Trans. Patt. Analysis Machine Intellig., 29: 915-928.

DOI: $10.1109 /$ TPAMI.2007.1110 